\title{
Diseño y validación de instrumento para la inserción del salario emocional ante la COVID-19
}

\section{Design and validation of an instrument for the insertion of emotional salary in the face of COVID-19}

Dra. Janeth Elizabeth Salvador-Moreno es directora de la Maestría de Seguridad y Salud Ocupacional de la Universidad San Gregorio de Portoviejo (Ecuador) (salvador.janeth@gmail.com) (http://orcid.org/0000-0003-4252-9271)

Mg. María Elena Torrens-Pérez es editora ejecutiva de Revista San Gregorio de la Universidad San Gregorio de Portoviejo Ecuador) (mariaelenatorrensperez@gmail.com) (https://orcid.org/0000-0003-3989-9765)

Dr. Vladimir Vega-Falcón es profesor e investigador de la Universidad Regional Autónoma de los Andes (Ecuador) (vega. vladimir@gmail.com) (https://orcid.org/0000-0003-0140-4018)

Dr. Darwin Raúl Noroña-Salcedo es profesor e investigador del Instituto Superior Tecnológico Sucre (Ecuador) (dnorona@ tecnologicosucre.edu.ec) (http://orcid.org/0000-0002-0630-0456)

\begin{abstract}
Resumen
El sector laboral se ha enfrentado responsablemente ante las afectaciones del coronavirus (COVID-19) y las adversidades que esta pandemia ocasiona, no obstante, este grupo humano no está excepto de riesgos y de afectaciones psicológicas por la gravedad de la situación en el ámbito laboral, que puede reasentar en la familia. Su actuación es consecuente con la realidad, aunque necesita de condiciones emocionales diferentes que le permitan continuar en su desempeño. La investigación tuvo como objetivos: diseñar un instrumento para medir el salario emocional y validar el instrumento creado para la inserción del salario emocional ante la COVID-19. La metodología empleada fue de tipo no experimental, descriptivo y transversal, y como variable de interés de estudio: el salario emocional. Se diseñó y validó un instrumento para medir el salario emocional, con un alfa de Cronbach de 0,925, el cual fue aplicado a una muestra de 207 trabajadores. Los encuestados manifestaron una predisposición favorable al instrumento y su percepción de salario emocional es considerada como alta. Como conclusiones se obtiene la reafirmación de la inserción del salario emocional, con estrategias de atención al recurso humano de forma personalizada, con respecto a sus emociones del ámbito laboral ante la escalada de la COVID-19.
\end{abstract}

\begin{abstract}
The labor sector has responsibly faced the effects of the coronavirus (COVID-19) and the adversities that this epidemic causes, however, this human group is not exempt from risks and psychological effects due to the seriousness of the situation in the workplace, which you can resettle into the family. His performance is consistent with reality, although he needs different emotional conditions that allow him to continue in his performance. The research had as objectives: to design an instrument to measure the emotional salary and validate the instrument created for the insertion of the emotional salary before COVID-19. The methodology used was non-experimental, descriptive, and transversal, and as a variable of study interest: the Emotional Salary. An instrument was designed and validated to measure emotional salary, with a Cronbach's alpha of 0.925, which was applied to a sample of 207 workers. Respondents expressed a favorable predisposition to the instrument and their perception of emotional salary is considered high. As conclusions, the reaffirmation of the insertion of the emotional salary is obtained, with strategies of attention to human resources in a personalized way, with respect to their emotions in the workplace in the face of COVID-19.
\end{abstract}

\section{Palabras clave I keywords}

Satisfactores, carga de trabajo, compensaciones, motivación del trabajador, salario emocional, ambiente de trabajo, retribuciones, validación de instrumento.

Satisfiers, workload, compensation, worker motivation, emotional salary, work environment, remuneration, instrument validation.

Cómo citar: Salvador-Moreno, J.E., Torrens-Pérez, M. E., Vega-Falcón, V., y Noroña-Salcedo, D.R. (2021). Diseño y validación de instrumento para la inserción del salario emocional ante la COVID-19. Retos Revista de Ciencias de la Administración y Economía, 11(21), pp. 41-52. https://doi. org/10.17163/ret.n21.2021.03 


\section{Introducción}

La sociedad en general, y el hombre en particular, se enfrenta a situaciones adversas para las cuales no está preparado, ante cada situación el trabajador se acopia de recursos propios de su naturaleza interior, por lo tanto, las respuestas no siempre se pueden medir en la misma magnitud, la resiliencia es inherente a la responsabilidad individual de cada persona. Así se vio representada la actuación y respuesta de cada profesional y trabajador ante la pandemia de la COVID-19, en todos los países e instituciones, con mayor rigor en el sector de la salud.

Todo el personal en general (administrativo, de la salud, operativo, de servicio, técnico, etc.), laboran en condiciones fuera de lo normal, para lo cual hay que hacer adaptaciones de protocolos, cambios de turnos, organización de equipos de trabajo, todo sobre la jornada de trabajo y de la adaptación de procesos (De Caneva et al., 2020). Para estas adaptaciones se requiere de líderes flexibles y motivadores, que depositen confianza en el hombre (Fundación más Humano, 2020) y las características que mayormente se manifiestan son las de cooperación y sensibilidad humana, todos cuidan de su vida, y de su familia.

En el Informe Especial de la CEPAL No. 3 (CEPAL, 2020) para América Latina, concluye en que la COVID-19 transcurre en momentos de crisis, lo que implica que la pobreza continúa y que las mayores consecuencias se darán en México, Nicaragua y Ecuador. Se reconoce además que "El mundo se enfrenta a una crisis sanitaria y humanitaria sin precedentes en el último siglo ... esta no es una crisis financiera sino de personas, de salud y bienestar" (Bárcena, 2020, diap.6), pues las cifras de contagios y de fallecidos, no son números, están relacionadas con las personas y hay que actuar, es necesario brindarles beneficios a los trabajadores que están inmersos en el contagio, para retribuirle en alguna medida la satisfacción y el reconocimiento que merece, por todo lo que entregan a la sociedad.

En la historia de enfermedades contagiosas y de pandemias, la COVID-19 se transmite con grandes afectaciones e impacto en la sociedad. Para enfrentar esta crisis el trabajo en equipo ofrece resultados alentadores, donde prima el esfuerzo de todos, unos separados por el distanciamiento, y otros creando límites para evitar el contagio, pero con la voluntad se aúnan fuerzas y compromisos.

En el contexto local, cuando en el Ecuador se registraron los 58 casos confirmatorios de COVID-19, se puso en práctica una serie de estrategias para disminuir la probabilidad de transmisión como fueron restricciones a espacios públicos, fortalecimiento de servicios hospitalarios, apertura al teletrabajo como modalidad laboral única durante el confinamiento, y cierre de fronteras. Con el paso de los meses las restricciones fueron acortándose en virtud de la urgencia de reactivar la economía, que golpeó fuertemente el costo de vida de la ciudadanía en general. No obstante, el panorama laboral nunca fue el mismo debido a una sobreexposición de factores de riesgo a la salud de los trabajadores (Ortega, 2020). Por lo que el desempeño laboral es decisivo ante la crisis sanitaria de la COVID-19 (Puitiza et al. 2020); y el reconocimiento al trabajador por su desempeño debe formar parte del ambiente de trabajo, con condiciones favorables que, además, reconozcan la participación del trabajador como una necesidad humana Vásquez et al. (2019).

Considerando además que, "El mercado laboral, es y ha sido el medio para sostener la vida de las personas, de sus familiares y del desarrollo social" (Cedeño \& Chávez, 2020, p. 145), el trabajo es vital para el hombre (Torrens \& Real, 2019). No obstante, en la actualidad el salario o remuneración no es suficiente (Hernández \& Osorio, 2016), ya que los empleados tienen percepciones y necesidades diferentes. El trabajador se pre- 
para y crece en experiencias, lo que le hace reaccionar diferente al trabajador de años anteriores, según Gómez (2011, p. 5) "por eso la importancia de tener una estrategia que ayude a la satisfacción del personal en su desempeño laboral".

Actualmente, el salario o remuneración económica no es el único indicador utilizado por la competencia laboral, pues ha dejado de ser decisivo, los empleados para su permanencia y satisfacción se enfocan en otros tipos de retribuciones como lo constituyen los beneficios que representen mayor satisfacción, no solo en lo profesional, sino que abarque mucho más. Para el hombre actual es importante satisfacer las necesidades personales y familiares, a lo que los autores se refieren como beneficios emocionales, con gran aporte en la calidad de vida (Howatt, 2011; Muñoz, 2011).

El trabajador manifiesta percepciones con respecto a su trabajo, su diferencia está dada por la naturaleza y variedad de necesidades de cada trabajador, teniendo en cuenta sus expectativas y aspiraciones, que están influenciadas por su cultura y personalidad, y se refieren a las expectativas que tiene cada trabajador con su futuro. Con respecto a esto Gómez (2011) manifiesta lo importante que resulta: "tener una estrategia que ayude a la satisfacción del personal en su desempeño laboral” (p. 5).

En las relaciones laborales entre el empleado y el empleador las compensaciones poseen gran importancia y pueden influir en otros indicadores de la organización, por lo que la compensación es determinante en el nivel de satisfacción laboral de un empleado (Mabaso \& Dlamini, 2017), estas actuaciones han sido aplicadas tanto en las empresas públicas, como en las privadas de Sudáfrica.

En el caso de México, existe la norma NOM-035-STPS-2018, (aplicada a partir de octubre del 2019), nombrada como "antiestrés" (Cázares, 2020), cuya aplicación en las empresas es de carácter obligatorio y su principal objetivo es que las entidades dispongan de entornos laborales beneficiosos para el trabajador, teniendo en cuenta la prevención de riesgos psicosociales en el ambiente de trabajo. Para la autora Cázares-Sánchez (2020) existe gran preocupación con respecto al cumplimiento de la norma, donde plantea que la aplicación de esta "implica el diseño de una estrategia de salarios emocionales en la que se haga distinción de la exigencia emocional de cada trabajo basada en un estudio, caso por caso" (p. 602), aspectos que particularizan el actuar de cada organización de acuerdo con sus características y necesidades internas del ambiente de trabajo y de las necesidades y percepciones de sus trabajadores. En México específicamente esta política no ha sido efectiva y esto puede estar dado porque el salario emocional, como concepto es poco difundido, como refieren Quintero y Betancur (2018) y Rubio et al. (2020).

En el caso de España se aplica el salario emocional en la escalada de la COVID19 por gerentes de Recursos Humanos de diferentes sectores, es una representación de lo que realmente debiera brindarse en tiempos de crisis sanitaria (Fundación más Humano, 2020; Emotional Salary Barometer, s.f.) y en estas actuaciones, los gerentes españoles han ofrecido un carácter más humano en el ámbito laboral.

\subsection{Salario emocional}

Atendiendo a los estudios consultados, la primera definición de salario emocional es a partir del año 2003, las investigaciones anteriores contemplan otras dimensiones como las compensaciones y retribuciones al trabajador. Para Huete (2003) "La capacidad de conseguir que las personas se sientan bien pagadas por su esfuerzo, con algo más que dinero" (p. 156). En cambio, para Gay (2006) es una forma de retribuir al trabajador, no monetaria, por el trabajo desempeñado, mientras que para Temple (2007) y Melo et al. (2018) son las razones no monetarias las que hacen que un trabajador desarrolle sus actividades contento y se sienta comprometido con la organización. Autores 
como Rocco (2009) consideran que son todos los factores que no se relacionan con la mensualidad económica y que aseguran su permanencia, además, de darle mayor satisfacción. Por otra parte Jiménez (2009) le agrega a lo anteriormente enunciado: que estas retribuciones le proporcionan al trabajador tranquilidad y desempeño eficaz y efectivo y que además deben permitir el trabajo en equipo, para lo cual se requiere de un liderazgo cooperativo. En este caso Gómez (2011) hace más énfasis en que las retribuciones deben estar encaminadas a la satisfacción de las necesidades afectivas y psicosociales de los trabajadores, que influyen en su bienestar emocional. También considera su repercusión favorable en la productividad y competitividad de la empresa. Es definido por Montalvo-Poveda (2018) como los beneficios intangibles que recibe el trabajador y su objetivo es el de complementar el salario económico y generar el compromiso del trabajador hacia su organización. Para Betancur y Quintero (2018) el salario emocional debe formar parte de la planeación estratégica, como uno de los objetivos organizacionales que a su vez motiven al trabajador.

Finalmente, los autores de la investigación declaran al salario emocional como los factores y satisfactores que logran bienestar emocional en el trabajador y permiten su desarrollo profesional y familiar, además de considerar que el salario emocional debe ser analizado por los empleadores y responsables para su implementación, toda vez que puede responder a las múltiples incógnitas de ¿cómo resolver la insatisfacción de sus empleados con respecto al trabajo que desempeñan?, debiendo ser constituido a partir de las particularidades y flexibilidades (Guzmán \& Del Pilar, 2018) sin que implique gastos monetarios (Rocco, 2009), argumentan además, que el salario emocional se corresponde con acciones creativas y particularizadas que le dan satisfacción a cada trabajador.

Para Minchán-Rojas (2017) el salario emocional tiene impacto sobre el trabajador de forma profesional, por lo que hay que tenerlo en cuenta en las compensaciones, tanto en el ambiente de trabajo como en lo personal, además recomienda que estos beneficios deben "ser tomados y enfrentados con instrumentos académicos y profesionales multidisciplinarios relacionados a la gestión del talento humano a nivel institucional" (p. 18).

Por lo que ante esta situación generada por la emergencia sanitaria de la COVID19, las instituciones públicas y privadas, independientemente del tipo de empresa y del sector que representan deben emplear formas diferentes para equilibrar el estado emocional de sus trabajadores, así como su satisfacción por el desempeño de su actividad y de su organización.

Los autores y las autoras declaran como objetivos para esta investigación: diseñar un instrumento para medir el salario emocional y validar el instrumento creado para la inserción del salario emocional ante la COVID-19.

\section{Materiales y método}

El estudio es de enfoque cuantitativo, con diseño no experimental y transversal, cuya primera etapa fue desarrollada en el período comprendido entre abril y agosto de 2020.

La variable de interés de estudio fue el salario emocional y como variables de caracterización se tuvieron en cuenta la edad, el género y la antigüedad en la institución, las cuales permitieron caracterizar a la población.

Se diseñó un instrumento para el contexto ecuatoriano, con base en la experticia de los autores y las autoras con más de veinte años relacionados con los temas de recursos humanos, psicología organizacional y administración de empresas. 


\subsection{Construcción del instrumento}

Durante el último semestre de 2020, se efectuaron cuatro actividades que permitieron la validación del instrumento de salario emocional. La primera fue la validación de contenido en la cual se investigaron las definiciones operacionales y conceptuales de la motivación, abarcando desde las definiciones de la equidad, justicia laboral y percepción del trabajador. Con base en las teorías de (Adams, Grifol \& Siegrist, 1962; Littlewood-Zimmerman \& Uribe-Prado, 2018).

Así también, para complementar de mejor manera la comprensión del fenómeno, se exploró el concepto mediante la entrevista a profundidad de expertos del área de talento humano, teniendo como pregunta inicial: ¿Cómo se puede medir el salario emocional? Durante la entrevista a profundidad, se anotaron las palabras clave que ayudaron a definir el concepto de salario emocional, grabándose las entrevistas en audio para posteriormente ubicar las palabras clave que permitieron agrupar los conceptos.

Con base en las actividades anteriormente citadas, se establecieron tres dimensiones que explican la variable del presente estudio: el ambiente, el desarrollo profesional y la flexibilidad.

En este sentido, en el ambiente laboral se incluyeron afirmaciones acerca de las condiciones gratificantes por parte del empleador y relacionados con criterios de confort como la cultura, el clima, capacitaciones y la preocupación por el colaborador. En el desarrollo profesional, se plantearon preguntas acerca de la estimación por las capacitaciones y la retroalimentación positiva por parte de los jefes. Finalmente, para el caso de flexibilidad, se situaron aspectos organizacionales en relación con la flexibilización y licencias. Todas las preguntas fueron redactadas en oración afirmativa. De las 37 preguntas iniciales, se confirmaron únicamente 18 afirmaciones, después de una depuración y análisis posterior.

El listado de ítems fue elaborado a través de la escala de Likert, cuyas respuestas fueron las siguientes 1: nunca; 2: casi nunca; 3: algunas veces; 4: frecuentemente; 5: casi siempre y 6: siempre. El listado final, fue aprobado a través de juicio de expertos constituido por tres (3) profesionales con Maestría en Gestión de Talento Humano quienes dieron la confirmación de la relevancia, suficiencia, claridad en la redacción.

La segunda y tercera actividad fueron la validación de constructo y cálculo de fiabilidad en una prueba piloto. La técnica utilizada fue la metodología de Nunnally (1978) que indica que por cada ítem se deberá validar a cinco sujetos. En tal virtud, el test fue aplicado a 300 trabajadores de una entidad gubernamental.

La prueba piloto, se realizó en el sector público ecuatoriano de la provincia de Manabí. La entidad cuenta con una población de 300 trabajadores empleando además los siguientes criterios de selección: se incluyeron a los trabajadores de más de seis meses, se excluyeron a quienes estaban en período de licencias y se eliminaron a los colaboradores que entregaron incompleto el instrumento. Sin embargo, solo 207 de las encuestas entregadas pudieron ser incluidas en el estudio por presentarse errores como dobles respuestas o falta de ellas, que invalidaron las restantes. Se realizó un cronograma de reuniones para las convocatorias de los trabajadores, que contempló áreas y turnos, en los que se les brindó información sobre la investigación a realizarse, el objeto de estudio, el contenido de la encuesta, la importancia de la honestidad en las respuestas, el anonimato del estudio y la forma de valoración. La explicación se complementaba con contenidos de utilidad para los trabajadores a fin de dar valor agregado para la entidad y no únicamente para el evaluador.

Para confirmar la proporción de la variabilidad de los resultados se utilizó el Alpha de Cronbach y al ratificar un valor de alta fiabilidad, se realizó un análisis factorial para determinar los grupos de ítems en sus dimensiones definitivas a través de la 
reducción de dimensiones, con solución inicial, prueba KMO de esfericidad de Barlett y rotación Varimax.

Finalmente, para el cálculo de los baremos se identificaron los percentiles 33 y 66 para contar con la significancia de percepción bajo, medio y alto de salario emocional.

Los trabajadores dieron su consentimiento de participación y se respetaron los principios éticos relacionados con la administración y utilización de la información acopiada, así como con la Declaración de Helsinki de la Asociación Médica Mundial, incluyendo sus actualizaciones (Asociación Médica Mundial, s.f.).

\section{Resultados}

Se encuestaron a un total de 207 trabajadores, de estos 186 son de género masculino $(89,9 \%)$ y 21 de género femenino $(10,1 \%)$. La edad del grupo femenino está entre 16 y 44 años, y la de los hombres entre 16 y 65.

Las variables sociodemográficas se muestran en la Tabla 1.

Tabla 1. Variables sociodemográficas

\begin{tabular}{|c|c|c|}
\hline Edad & Frecuencia & Porcentaje \\
\hline $16-24$ & 18 & 8,7 \\
\hline $25-34$ & 119 & 57,5 \\
\hline $35-44$ & 47 & 22,7 \\
\hline $45-54$ & 22 & 10,6 \\
\hline $65-$ o más & 1 &, 5 \\
\hline Total & 207 & 100,0 \\
\hline Género & Frecuencia & Porcentaje \\
\hline Masculino & 186 & 89,9 \\
\hline Femenino & 21 & 10,1 \\
\hline Total & 207 & 100,0 \\
\hline Antigüedad & Frecuencia & Porcentaje \\
\hline $1-5$ & 42 & 20,3 \\
\hline $6-10$ & 77 & 37,2 \\
\hline $11-15$ & 33 & 15,9 \\
\hline $16-20$ & 24 & 11,6 \\
\hline $21-25$ & 15 & 7,2 \\
\hline $26-30$ & 16 & 7,7 \\
\hline Total & 207 & 100,0 \\
\hline
\end{tabular}

Fuente: Elaboración propia.

\subsection{Depuración de los ítems y consistencia interna}

La encuesta de salario emocional estuvo finalmente constituida por 18 ítems, conteniendo tres factores (factor 1, factor 2, y factor 3) con un alfa de Cronbach de 0,925.

Las matrices de factores rotados mostraron tres factores que explicaron el 60,12\% de la varianza acumulada. Estos factores y sus preguntas se muestran en la Tabla 2. 


\section{Tabla 2. Factores del instrumento encuestan salario emocional}

\begin{tabular}{|c|c|c|}
\hline Dimensión & \# Preg. & Pregunta \\
\hline \multirow{6}{*}{ Factor 1} & 1 & Los jefes o directivos acostumbran a saludar al personal \\
\hline & 4 & $\begin{array}{l}\text { La empresa le brinda capacitaciones que luego aplicas en tu trabajo o } \\
\text { en tu vida personal }\end{array}$ \\
\hline & 5 & Has desarrollado amistad con tus compañeros de trabajo \\
\hline & 7 & $\begin{array}{l}\text { Cuando alguien tiene algún problema familiar (enfermedad o sinies- } \\
\text { tro), tus superiores se solidarizan y ayudan }\end{array}$ \\
\hline & 12 & Te sientes alegre de ser parte de esta empresa \\
\hline & 17 & $\begin{array}{l}\text { Cuando despiertas te agrada la idea de ir a tu trabajo y reunirte con } \\
\text { tus compañeros }\end{array}$ \\
\hline \multirow{6}{*}{ Factor 2} & 2 & $\begin{array}{l}\text { Les brindan capacitaciones que luego aplicas en tu trabajo o en tu } \\
\text { vida personal }\end{array}$ \\
\hline & 8 & Existen posibilidades de ascenso por méritos \\
\hline & 10 & $\begin{array}{l}\text { Tus opiniones, informes o sugerencias son bien recibidas por tus su- } \\
\text { periores }\end{array}$ \\
\hline & 13 & $\begin{array}{l}\text { Reconocen con gestos, frases o felicitaciones cuando cumplen a caba- } \\
\text { lidad con alguna tarea especial }\end{array}$ \\
\hline & 16 & $\begin{array}{l}\text { Te toman en cuenta para actividades, trabajos o comisiones especiales } \\
\text { que debe cumplir la empresa }\end{array}$ \\
\hline & 18 & Se presentará la renuncia crees que te pedirían que te quedes \\
\hline \multirow{6}{*}{ Factor 3} & 3 & $\begin{array}{l}\text { Se puede pedir permisos para trámites bancarios, legales o de índole } \\
\text { personal }\end{array}$ \\
\hline & 6 & $\begin{array}{l}\text { Desarrollan actividades deportivas o de recreación con todo el perso- } \\
\text { nal }\end{array}$ \\
\hline & 9 & Tienes la oportunidad de alimentarte durante tu jornada de trabajo \\
\hline & 11 & $\begin{array}{l}\text { Puedes contar con tus descansos (fines de semana, feriados o días } \\
\text { libres) sin que te interrumpan o te llamen del trabajo. }\end{array}$ \\
\hline & 14 & $\begin{array}{l}\text { Tienes espacios para compartir un café o una broma con tus compa- } \\
\text { ñeros de trabajo }\end{array}$ \\
\hline & 15 & $\begin{array}{l}\text { Te brindan alguna flexibilidad en horarios si tuvieras una emergencia } \\
\text { o quisieras estudiar. }\end{array}$ \\
\hline
\end{tabular}

Fuente: Elaboración propia

\subsection{Criterio de consistencia interna}

Los valores de fiabilidad se muestran en la Tabla 3 para cada uno de los factores finales del instrumento.

Tabla 3. Valores del alfa de Cronbach por variable

\begin{tabular}{|l|l|l|}
\hline \multicolumn{1}{c|}{ Variables } & No items inicial & \multicolumn{1}{c|}{ Final $\alpha$} \\
\hline Factor 1 & 6 & 0,97 \\
\hline Factor 2 & 6 & 0.96 \\
\hline Factor 3 & 6 & 0.98 \\
\hline
\end{tabular}

Fuente: Elaboración propia. 


\subsection{Escala de calificación del instrumento}

Para la calificación de la escala se procedió a calcular los percentiles 33 y 66 de los resultados de los 18 ítems del instrumento con base en las tres posibles respuestas. Se obtienen tres escalas, tal como se muestra en la Tabla 4.

Tabla 4. Escala de calificación del instrumento

\begin{tabular}{|l|l|l|l|}
\multicolumn{1}{|c|}{ Variables } & \multicolumn{1}{c|}{ Bajo } & \multicolumn{1}{c|}{ Medio } \\
\hline Factor 1 & $6-25$ & $26-29$ & $30-36$ \\
\hline Factor 2 & $6-19$ & $20-25$ & $26-36$ \\
\hline Factor 3 & $6-22$ & $23-27$ & $28-36$ \\
\hline TOTAL & $6-69$ & $70-83$ & $84-108$ \\
\hline
\end{tabular}

Fuente: Elaboración propia.

La respuesta del personal encuestado arrojó resultados que bien pudiesen calificarse como sorpresivos, al mostrarnos su posición frente a las compensaciones no monetarias recibidas en su sitio de trabajo, toda vez que los resultados fueron positivos.

En el salario emocional se ha considerado tres dimensiones: el ambiente, el desarrollo y la flexibilidad, además del valor global del salario emocional como tal y se puede notar algunos valores relevantes que permiten la orientación hacia circunstancias que las personas consideran más valiosas o de significativa importancia.

El primer análisis revisado son los resultados por el género de los participantes en la investigación, debiendo indicar que, de las 207 encuestas válidas tabuladas, el 89,9\% corresponden al género masculino y el 10,1\% al género femenino.

Para poder interpretar los resultados, se establecieron parámetros guía, de forma que aquellos resultados que oscilan entre 1 y 12 corresponden a una calificación BAJA, los que oscilan entre 13 y 24 una calificación MEDIA y los que van de 25 a 36 ALTA.

\section{Figura 1. Salario emocional por género}

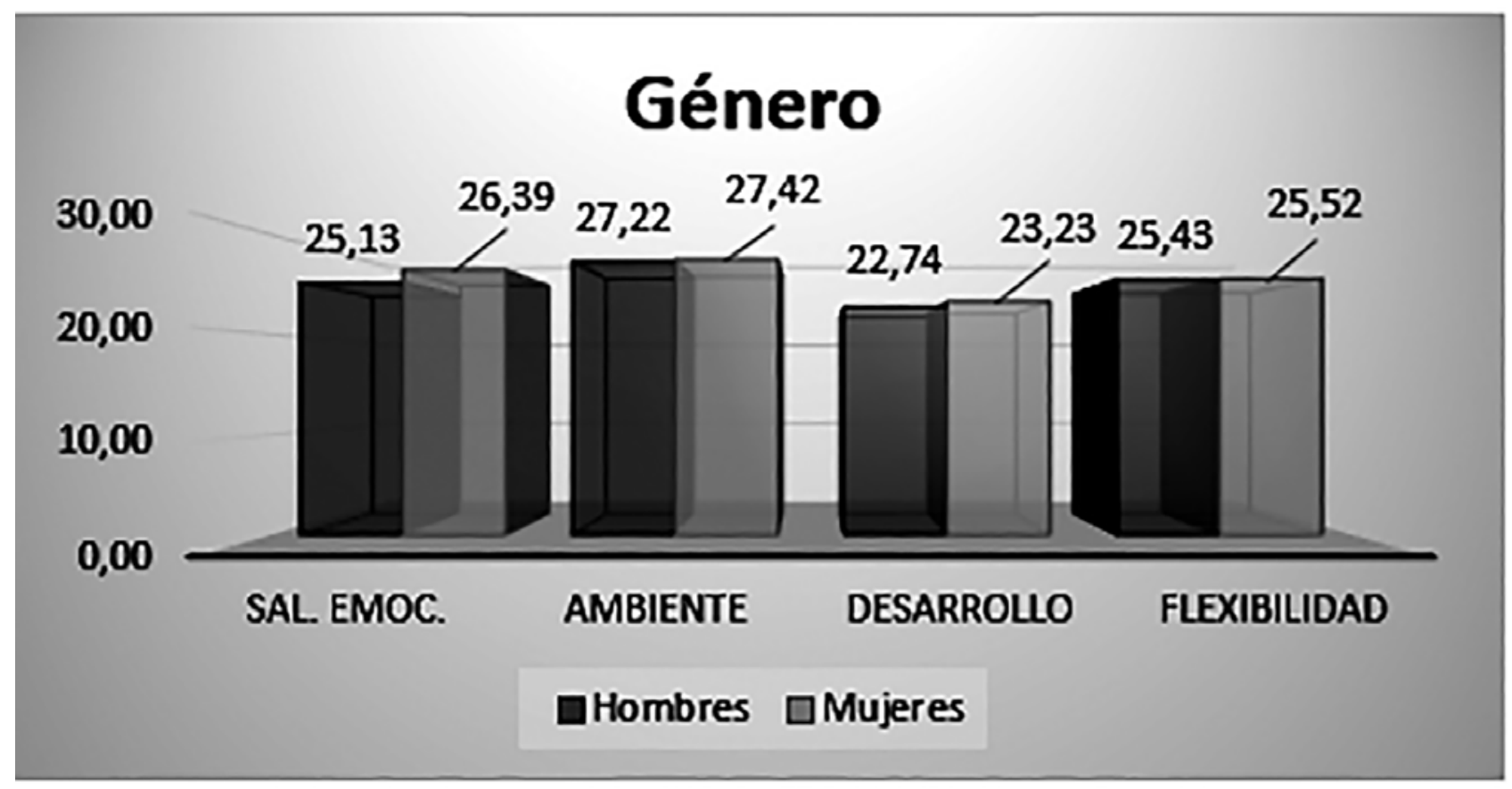

Fuente: Elaboración propia. 
Como se puede notar en la Figura 1, la percepción de las compensaciones no monetarias o salario emocional del personal tanto femenino como masculino es alto y las mujeres le han dado un mayor valor, aunque con una diferencia no muy marcada en relación con lo que consideran los hombres.

$\mathrm{Al}$ momento de hacer un desglose de las dimensiones, se puede notar una ligera diferencia entre los géneros, siendo las mujeres las que presentan el porcentaje más elevado en todas ellas.

Cuando se hizo la revisión de los resultados por cada pregunta, se puede destacar que el $89 \%$ de los encuestados están de acuerdo y totalmente de acuerdo, en que sus sugerencias, reportes u opiniones, son bien recibidas por sus superiores; de igual manera un $79 \%$ manifiesta sentirse feliz de ser parte de la empresa, la primera apreciación corresponde a la dimensión de desarrollo y la segunda a la dimensión ambiente. En la dimensión flexibilidad el 64\% está de acuerdo y/o totalmente de acuerdo en que se les brinda un espacio para tomar sus alimentos; y, un 59\% indican que la empresa les permite abandonar sus labores para realizar trámites personales. Los resultados nos dan una clara orientación a situaciones en los que la empresa ha logrado generar respuestas positivas en su personal, sin costo o con un costo de bajo impacto.

También existen los casos opuestos, es decir, aquellas preguntas donde el porcentaje de respuesta fue altamente desfavorable, de ahí que el $42 \%$ contestó que está en "desacuerdo" y "totalmente en desacuerdo" en que existen posibilidades de ascenso por méritos, lo que debería despertar las alarmas institucionales y revisar lo que origina esta percepción en su personal. Otro planteamiento que generó un 30\% de percepción negativa, es la relacionada a la posibilidad que sus jefes les pidieran permanecer en su trabajo ante una renuncia, sobre el particular debo indicar que solo un 38\% dio respuesta positiva y el restante $32 \%$ está en ese intermedio que también debe considerarse negativo.

En cuanto a la dirección que toma el salario emocional en relación con el tiempo de permanencia en la actividad, aquellos profesionales que tenían más de 25 años de servicio, reflejan el índice más alto de salario emocional pero solo representan el 7\% de los encuestados, les sigue el grupo de trabajadores que tienen entre 16 a 20 años de trabajo y representan al 9\% de los encuestados, ambos grupos presentan un alto nivel de salario emocional. El grupo de trabajadores con uno a cinco años, representan al $20,38 \%$ de los encuestados y muestran un salario emocional alto $(25,64)$ y el grupo de trabajadores de 21 a 25 años de servicio, (7\% de los encuestados) se presentan con el índice más bajo de salario emocional $(22,31)$.

\section{Discusión y conclusiones}

El valor del alfa de Crombach obtenido en el análisis de fiabilidad del instrumento corrobora que los factores, el número de los ítems y la redacción de las preguntas no interfieren en el error de varianza de los sujetos encuestados. Al respecto Oviedo y Arias (2005) indican que valores de fiabilidad por encima del 0,80 garantizan la aplicación de instrumentos consistentes y válidos.

Por otra parte, el análisis factorial confirmatorio realizado con los datos de esta encuesta, reveló la presencia de tres factores que explican más del $60 \%$ de la varianza total acumulada. No obstante, la disposición de los ítems en estos tres grupos no correspondió con la posición exacta de las preguntas en las categorías ambiente, desarrollo y flexibilidad. Con base en las consideraciones de (Lloret et al., 2014) los ítems de una escala se organizan de acuerdo con sus correlaciones altas entre sí, pero si bien el análisis factorial confirma la validez de constructo, es decisión del autor del instrumento adoptar el ordenamiento. Si, por ejemplo, los factores generados no guardan 
relación con las definiciones conceptuales y operacionales de las variables estudiadas, es mejor acomodar según el criterio teórico y volver a calcular el análisis confirmatorio con una segunda muestra poblacional.

Aliaga-Tovar (2007) en su obra acerca de los test psicométricos, recomienda que cuando se construyan las escalas, estas se deben dotar de sus respectivas validaciones a través del criterio de consistencia externa. En tal virtud, si bien la escala de salario emocional obtuvo valores significativos de confiabilidad, será necesario calcular la correlación estadística de sus resultados frente a otro instrumento que evalúe este fenómeno para asegurar la validez total de criterio.

La percepción de satisfacción alta de salario emocional de la investigación, se opone a la encontrada en las conclusiones de Chaparro-Espitia (2006). Los trabajadores valoran positivamente la parte económica y dejan a un lado estímulos como los incentivos, el reconocimiento o los permisos. En este estudio se valora más la fraternidad antes que los aspectos emocionales de la retribución de la empresa. En contraste, en la investigación de López-Más (2005) se recalca que los trabajadores, aun los que reciben menor remuneración, incorporan en su percepción de satisfacción los incentivos emocionales que no generan acumulación de capital directamente, pero que desarrollan sus competencias, conocimientos, y habilidades generando autoestima y sentimientos de auto eficiencia.

En la actualidad, no existen instrumentos cuantitativos reconocidos y específicos para medir la percepción de salario emocional. La gran mayoría de las encuestas de clima laboral, presentan dimensiones que describen la satisfacción que sienten los trabajadores hacia la remuneración. En este sentido, la encuesta de Gómez-Rada (2004), incorpora en el factor llamado apoyo, las retribuciones emocionales que la empresa brinda a sus trabajadores. El proceso de validación presenta acciones similares (validación de contenido, de criterio y de constructo), con un número de cinco veces más preguntas que la escala emocional de este estudio. El valor Alfa de Cronbach final fue de 0,84 con la depuración de 70 ítems. En cuanto al análisis factorial, se confirmaron cuatro factores que explicaron el $53,55 \%$ de la varianza. En este punto, los realizadores de este instrumento de clima laboral, tuvieron que readaptar las preguntas, puesto que los grupos factoriales no correspondían a la definición inicial.

En otros estudios de validación de instrumentos de clima laboral (Chiang-Vega et al., 2008; Arica-Rodríguez et al., 2015; Salaiza-Lizárraga \& Vela-Nava, 2008) se constata que el valor de alfa de Cronbach mantiene las puntuaciones de 0,8 a 0,9, con factorizaciones que no explican más allá del 50\% de la varianza total, y con un número similar de preguntas que responden al salario emocional en aspectos tales como el ambiente, el desarrollo y la flexibilidad. Sin embargo, hay que recalcar que la muestra para la construcción de estos predictores asciende a un número mucho mayor que el de la presente investigación. Esto permite comprender los registros de sus varianzas. Otra de las características que presentan estos instrumentos es la estandarización de su población, ya que los predictores están diseñados a grupos ocupacionales específicos como médicos, oficinistas o profesores con lo cual se asegura mayor precisión en los datos finales.

Las personas encuestadas mostraron satisfacción ante el grupo de preguntas y las consideraron pertinentes y ajustadas a la realidad de su entorno, sin embargo, el estudio aplicado solo demuestra que este grupo de profesionales cuenta con situaciones compensatorias que complementan a su salario monetario, pero no nos determina el nivel de influencia de estas compensaciones no monetarias, sobre su voluntad de permanencia en su sitio de trabajo, en su actividad laboral o profesional, ni la relación que guarda con el salario monetario. 
Al medir la variable de estudio de salario emocional con respecto al resto de las variables demográficas, se constata un ligero crecimiento en el valor puntuado por las mujeres, esta pequeña diferencia puede estar dada por la diversidad de las ocupaciones en las cuales se encuentran: ser responsables de familia (madre soltera), por los contratos por tiempo determinado, que le da inseguridad, aspectos que deben tenerse en cuenta para próximas investigaciones.

Bajo un análisis administrativo se podría aseverar que este grupo de profesionales, pese a lo adverso de las condiciones, han recibido un buen trato por parte de sus superiores los que en alguna medida les resulta no solo compensatorio, sino que además les permite mantener un cierto nivel de motivación para desarrollar sus labores de manera apropiada.

Esta primera publicación es el punto de partida para próximos estudios, en las que se pretenden continuar la línea de investigación, que permita analizar el ámbito laboral y al talento humano inmerso en las actuales condiciones, donde ocupe una dimensión diferente en la organización por su importancia, y por las contribuciones que desempeña, como refiere Chiavenato (2011). No es propicio continuar con estereotipos de otras décadas, que no reconozcan la valía del empleado y su influencia en los resultados finales de las organizaciones. Esta investigación surgió por la necesidad de reconocer la importancia del estado emocional del trabajador en su desempeño, no obstante, es necesaria una práctica más generalizada y una socialización del concepto y beneficios del salario emocional.

Los resultados presentados en el estudio, constituyen el preámbulo para posteriores investigaciones en diferentes sectores, en los que pueden ser incluidos indistintamente el sector público y el privado, con una muestra más amplia y con mayor diversidad por lo que se conformará un proyecto de investigación interinstitucional.

\section{Referencias}

Adams, J.S., \& Rosenbaum, W.B. (1962). The relationship of worker productivity to cognitive dissonance about wage inequities. Journal of Applied Psychology, 46(3), 161. https://doi.org/10.1037/h0047751

Aliaga-Tovar, J. (2007). Psicometría: Tests psicométricos, confiabilidad y validez. Psicología: Tópicos de actualidad, 85-108. https://bit.ly/3uIagAq

Arica-Rodríguez, L., Rojas-García, K., Iglesias-Curto, D., \& Caller-Chuecas, M. (2015). Construcción y validación de una herramienta que mida el clima laboral y la implementación de acciones que lo favorezcan. https://bit.ly/3uJSooL

Asociación Médica Mundial (s.f.). Declaración de Helsinki. Asociación Médica Mundial. https://bit.ly/3q5SVij.

Bárcena, A. (2020). Coyuntura, escenarios y proyecciones hacia 2030 ante la presente crisis de Covid-19 3 Secretaria Ejecutiva. Comisión Económica para América Latina y el Caribe (CEPAL). https://bit.ly/2OoLyEL.

Betancur, J.G., \& Quintero, V. (2018). Salario emocional y su influencia en la productividad de los colaboradores del área bóveda y custodia del conglomerado financiero Banco Popular y de Desarrollo Comunal, en el primer semestre del año.

Cázares-Sánchez, C. (2020). La inclusión del salario emocional en México Norma Oficial Mexicana NOM-035-STPS-2018. Factores de riesgo psicosocial en el trabajo- Identificación, análisis y prevención. Revista Cambios y Permanencias, 11(1), 602-624. https://bit.ly/3a9XGSB

Cedeño-Bravo, A.P., \& Chávez-Carrillo, R.A. (2020). Análisis comparativo de las herramientas ISTAS 21 y FPSICO en personal del sector financiero. Revista San Gregorio, 1(39), 143-161. https://bit.ly/380A7df

CEPAL (2020). El desafío social en tiempos del COVID-19. Informe Especial No. 3. ECLAC. 


\section{https://bit.ly/3b4ZGuD.}

Chaparro-Espitia, L. (2006). Motivación laboral y clima organizacional en empresas de telecomunicaciones: Factores diferenciadores entre las empresas pública y privada. Innovar, 16(28), 7-32. https://bit.ly/3sjoHsP.

Chiang-Vega, M.M., Salazar-Botello, C.M., Huerta-Rivera, P.C., \& Núñez-Partido, A. (2008). Clima organizacional y satisfacción laboral en organizaciones del sector estatal (Instituciones públicas) Desarrollo, adaptación y validación de instrumentos. Universum (Talca), 23(2), 66-85. http://doi.org/10.4067/S0718-23762008000200004

Chiavenato, I. (2011). Administración de recursos humanos. El capital humano de las organizaciones. Mc Graw Hill. Recuperado de https://bit.ly/3rHsSOD.

De Caneva, F., García-Gómez, M., \& Baur, E.B. (2020). Salud emocional del personal sanitario del Área de Urgencias durante la pandemia COVID-19. Medicina Clínica. https://doi.org/10.1016/j.medcli.2020.06.011

Emotional Salary Barometer (s.f.). Emotional Salary Barometer. https://bit.ly/3bAveIX.

Fundación más Humano (2020, julio 30). El salario emocional, un factor clave para las organizaciones en la crisis del Covid-19. https://bit.ly/3p6uJe8.

Gay, F.P. (2006). El salario emocional, clave para reducir el estrés. Gestión práctica de riesgos laborales: Integración y desarrollo de la gestión de la prevención, 33, 44-47. https://bit.ly/3uKyBFO

Gómez-Rada, C.A. (2004). Diseño construcción y validación de un instrumento que evalúa clima organizacional en empresas colombianas desde la teoría de respuesta al ítem. Acta colombiana de psicología, 11, 97-113. https://bit.ly/3dX5tFn

Gómez, C. (2011). El salario emocional (Borrador de administración No. 47). https://bit.ly/3uIg0dB

Guzmán, L., \& Del Pilar, R. (2018). El salario emocional, una estrategia para incrementar la retención del talento humano en el sector asegurador. (Tesis de grado). Universidad Militar Nueva Granada. https://bit.ly/3tHT7qb

Hernández-Ortiz, R.A., \& Osorio-Suárez, L.F. (2016). El salario emocional en la remuneración del talento humano de la generación del milenio en Colombia. (Tesis MBA Internacional). Colegio de Estudios superiores de Administración-CESA. https://bit.ly/2NnMgkR.

Howatt, W.A. (2011). Por qué el personal permanece y por qué se va: Cómo atraer y retener empleados talentosos. Panorama Editorial.

Huete, L.M. (2003). Servicios y beneficios. Deusto.

Jiménez, A. (2009). Gestión de personas y tendencias del mercado laboral. Harvard Deusto Business Review, 185, 45-51.

Littlewood-Zimmerman, H.F., \& Uribe-Prado, J.F. (2018). Psicología organizacional en Latinoamérica. Manual Moderno.

López-Más, J. (2005). Motivación laboral y gestión de recursos humanos en la teoría de Frederick Herzberg. Gestión en el tercer milenio, 8(15), 25-36. https://doi.org/10.15381/gtm.v8i15.9692

Lloret, S., Ferreres, A., Hernández, A., \& Tomás, I. (2014). El análisis factorial exploratorio de los ítems: Una guía práctica, revisada y actualizada. Anales de Psicología/Annals of Psychology, 30(3), 1151-1169. https://doi.org/10.6018/analesps.30.3.199361

Mabaso, C.M., \& Dlamini, B.I. (2017). Impact of Compensation and Benefits on Job Satisfaction. Research Journal of Business Management, 11(2), 80-90. https://doi.org/10.3923/rjbm.2017.80.90

Melo-Martínez, C., Prieto-Cagua, N.Y., \& Tarazona-Gómez, Y.C. (2018). Cornisa: Modelo de salario emocional para Cardiocolombia SAS. https://bit.ly/2ZdR66U.

Minchán-Rojas, O.L. (2017). Salario emocional y retención del talento humano en la Oficina General de Recursos Humanos del Ministerio de la Mujer y Poblaciones Vulnerables, 2017. (Tesis de grado académico de: Maestro en Gestión Pública). Escuela de Posgrado, Universidad Cesar Vallejo, Perú. https://bit.ly/379avud.

Montalvo-Poveda, M.E. (2018). Salario emocional y rotación de personal en la empresa Consulting and Tax. (Tesis de Grado). Universidad Central del Ecuador. https://bit.ly/3d5vW34.

Muñoz, L.A. (2011). Política retributiva flexible y modelo de retribución en la PYME. https://bit.ly/3aegjET.

Nunnally, J.C. (1978). Psychometric theory. McGraw-Hill. 
Ortega, M. (2020). Efectos del Covid-19 en el comportamiento del consumidor: Caso Ecuador. Retos. Revista de Ciencias de la Administración y Economía, 10(20), 233-247. https://doi.org/10.17163/ret.n20.2020.03.

Oviedo, H.C., \& Arias, A.C. (2005). Aproximación al uso del coeficiente alfa de Cronbach. Revista Colombiana de Psiquiatría, XXXIV(4), 572-580. https://bit.ly/3jGWVmW.

Puitiza-Lucano, C.M., Tejada-Muñoz, S., Morales-Rojas, E., Chávez-Quintana, S., \& Sánchez- Santillán, T. (2020). Clima organizacional y desempeño laboral en trabajadores de la microred de salud Bagua Grande en Perú //Organizational climate and work performance in workers of the Bagua Grande health microgrid in Peru. Revista de la Universidad del Zulia, 11(31), 71-85. https://doi.org/10.46925//rdluz.31.06

Quintero, L., \& Betancur, J. (2018). Modelo de salario emocional para la fidelización de los colaboradores en la búsqueda de una organización competitiva. Espacios, 39(41), 8-13.

Rocco-Cañón, M.T. (2009). Satisfacción laboral y salario emocional: Una aproximación teórica. (Tesis de grado). Universidad de Chile. https://bit.ly/3qjv0fw

Rubio, S.M., Beltrán, C.A., Baltazar, R.G., \& Gómez-Sánchez, R.V. (2020). El concepto de salario emocional. Revista Científica Retos de la Ciencia, 4(1), 15-24.

Salaiza-Lizárraga, F. de la C., \& Vela-Nava (2008). Validación de un instrumento para medir el clima laboral en instituciones educativas. Ponencia presentada en XII Congreso Anual de la Academia de Ciencias Administrativas AC (ACACIA), Monterrey. https://bit.ly/3pnh037

Temple, I. (2007, agosto 23). El salario emocional. El Comercio.pe. https://bit.ly/3794uxD

Torrens-Pérez, M.E., \& Real-Goya, G. (2019). Salarios Mínimos y Necesidades Vitales. ECA Sinergia, 10(3), 108-118. https://doi.org/10.33936/eca_sinergia.v10i3.1983

Vásquez-Gastelumendi, D.V., Ríos-Campos, C.A., Santamaría-Baldera, N., Gutiérrez-Valverde, K.S., Camacho-Delgado, F.M., Aguirre-Zaquinaula, I.R., \& Estela Urbina, R.O. (2019). Estrategias organizacionales para fortalecer el clima laboral en la Escuela Profesional de Tecnología Médica-Facultad de Ciencias de la Salud de la Universidad de Chiclayo. Revista de la Universidad del Zulia, 10(28), 112-136. https://bit.ly/3dCno4h 